\title{
Anti-CD33/CD3 Bispecific Antibody GEM 333
}

National Cancer Institute

\section{Source}

National Cancer Institute. Anti-CD33/CD3 Bispecific Antibody GEM 333. NCI Thesaurus. Code C155970.

A bispecific antibody possessing two antigen-recognition and binding sites, one for the CD3 complex, a group of T-cell surface glycoproteins that complex with the T-cell receptor (TCR), and one for the tumor-associated antigen (TAA) CD33, with potential immunostimulating and antineoplastic activities. Upon administration of anti-CD33/CD3 bispecific antibody GEM 333, this bispecific antibody binds to both the CD3 antigen on cytotoxic T-lymphocytes (CT Ls) and the CD33 antigen found on CD33-expressing tumor cells, thereby crosslinking tumor cells and CT Ls. This activates and redirects CT Ls to CD33-expressing tumor cells, which results in the CT L-mediated cell death of CD33expressing tumor cells. CD33, a myeloid differentiation antigen, is expressed on normal non-pluripotent hematopoietic stem and progenitor cells (HSPCs) and is overexpressed on a variety of cancer cell types, including acute myeloid leukemia (AML). It plays a key role in tumor initiation, proliferation and progression. 